\title{
Nueva metodología para valorar la calidad de las aguas superficiales para su uso como clase 2 en Costa Rica
}

\author{
New methodology for evaluating the surface waters \\ quality to be used as Class 2 in Costa Rica
}

Guillermo Calvo-Brenes'

Fecha de recepción: 9 de agosto del 2012 Fecha de aprobación: 14 de noviembre del 2012

Calvo-Brenes G. Nueva metodología para valorar la calidad de las aguas superficiales para su uso como clase 2 en Costa Rica. Tecnología en

Marcha. Vol. 26, N². Pág 9-19

Químico. Estudiante Doctorado en Ciencias Naturales para el Desarrollo (DOCINADE) e Investigador del Centro de Investigación en Protección Ambiental (CIPA), Escuela de Química del Instituto Tecnológico de Costa Rica, Cartago. Teléfono 25502807. Correo electrónico : gcalvo@itcr.ac.cr. 


\section{Palabras clave}

Indice de calidad del agua; indicadores de calidad del agua; recurso hídrico; contaminación de ríos; Índice Holandés de clasificación de la calidad del agua

\section{Resumen}

Para determinar la calidad de las aguas superficiales se utilizan distintos indicadores físicoquímicos, microbiológicos y biológicos, así como índices de calidad. El reglamento costarricense contempla el uso de dos índices: uno se basa en la determinación de la fauna bentónica y el otro es el Indice Holandés. Este último requiere únicamente el análisis de tres indicadores físicoquímicos de calidad, lo cual puede generar algún grado de ambigüedad y falta de robustez.

Es por ello que es conveniente no descartar el uso de otros índices recomendados en la literatura. La mayoría de ellos requiere la transformación de los indicadores en valores adimensionados (SI) en una escala de 0 a I00. En esta investigación, para el cálculo del SI se utilizaron las fórmulas mencionadas por Cude, Nasirian, Dinius, Prakash, Prati, Walski-Parker y Stoner. Los distintos SI así generados deben mostrar un comportamiento que varíe en una escala de 0 a 100, lo cual a su vez también debe concordar con los valores de permisibilidad reportados en nuestro reglamento para cada indicador. En general, a mayor concentración de un indicador en particular, mayor la tendencia del SI hacia 0, lo que representa un mayor grado de contaminación de los ríos.

El objetivo de esta investigación fue verificar la aplicabilidad de dichas fórmulas matemáticas para la obtención del SI y su posible aplicación en Costa Rica, considerando nuestro entorno ambiental y nuestra reglamentación. La aplicación se hizo específicamente para la clase 2 mencionada en el reglamento costarricense.

Al analizar las distintas fórmulas de cálculo propuestas por varios investigadores, se encontró que la mayoría son apropiadas para las zonas a las que hacen referencia sus autores y en general son diferentes de nuestro entorno ambiental. También, algunas de ellas son para usos específicos de las aguas superficiales y además los valores de permisibilidad empleados difieren de los indicados por nuestra legislación, lo cual hace inviable su uso en nuestro país. Fue necesario, por tanto, proponer nuevas fórmulas de cálculo para la determinación del SI que sean aplicables a Costa Rica y acordes con su legislación. Fue necesario también proponer una nueva categorización de la calidad del agua clase 2 que, además, se puede utilizar con cualquier índice que se desee evaluar.

\section{Key words}

Water quality index; water quality variable; hydric resources; river contamination; water quality classification Holland index.

\begin{abstract}
The surface water quality determination is based through the analysis of a fair amount of physic chemicals, microbiological and biological water quality variables, as well as water quality indexes. The Costa Rican regulation considers the use of two indexes: one based on benthic invertebrate analysis and the other on the Water Quality Holland Classification Index. The last one requires only three physic chemical variables that could generate some ambiguity and lack of strength in that index.

It is therefore recommended not to discard the use of other indexes mentioned in the literature. Most of them require transforming the unit's variable as dimensionless $(\mathrm{SI})$ in a 0-100 scale. For the SI calculation, the Cude, Nasiriam, Dinius, Prakash, Prati, Walski-Parker and Stoner formulas were used. The $\mathrm{SI}$ should show a 0-I00 scale variation. As a rule, the more concentration of a variable, the more tendency to 0 of the $\mathrm{Sl}$, which it is expected to have a close relationship with Costa Rican regulations.
\end{abstract}

The objective of this research was to verify the aplicability of calculation formulas in order to obtain the $\mathrm{SI}$ and its posible aplication in our country, considering our own environment and regulations. All the analysis were focused on Class 2 mentioned in our national regulation.

After the analysis of different formulas proposed by researchers, it was found that most of them were appropiate for their own environment but not for ours. Some are appropiate for different types of waters and their regulations are different for our country. It was necessary to propose new ways of 
calculating the SI for our country and to propose a new way of categorizing the quality of the Class 2 that is also useful for the aplication of several indexes.

\section{Introducción}

En Costa Rica, los criterios y metodologías que se utilizan para evaluar la calidad de los cuerpos de agua superficiales están establecidos en el "Reglamento para la evaluación y clasificación de la calidad de cuerpos de agua superficiales" (MINAE, 2007). Esta normativa pretende que los criterios de calidad establecidos permitan la clasificación de los ríos para los diferentes usos que puedan darse a este bien. Se hace referencia a II distintos usos del agua que, a su vez, se agrupan en cinco clases. De esta forma, es factible que una clase particular presente distintos usos del agua (ver Cuadro I). Dicha clasificación por clases estará condicionada a los valores de permisibilidad establecidos para los indicadores físicoquímicos, microbiológicos y biológicos indicados en el citado reglamento. Para mayores detalles, se recomienda la lectura del reglamento.

Los indicadores son datos estadísticos o mediciones de una cierta condición, cambio de calidad o cambio de estado de lo que se evalúa. La determinación de la calidad de un cuerpo de agua usualmente conlleva el análisis de una cantidad grande de indicadores, los cuales se expresan en diferentes magnitudes 0 unidades y tienen diferente comportamiento en términos de su relación concentración-impacto (Abbasi, 2002, Department of Environmental Conservation, 201I, Enderlein, 2010, EPA, 1988, EPA, 2008, EPA, 2012. Natural Resources Board, 201I, Parparov, 2006). Si varios indicadores se integran de forma que generen un solo valor, se les denomina índices (CEPIS, 2008, FAO, 200I). Un índice se usa para sintetizar una gran cantidad de información de

Cuadro I. Clasificación de los cuerpos de agua, según el uso potencial y el tratamiento que requieran

\begin{tabular}{|c|c|c|c|c|c|}
\hline Usos \Categorías & Clase I & Clase 2 & Clase 3 & Clase 4 & Clase 5 \\
\hline $\begin{array}{l}\text { Abastecimiento de agua para uso y consumo } \\
\text { humano }\end{array}$ & $\begin{array}{l}\text { Con } \\
\text { tratamiento } \\
\text { simple con } \\
\text { desinfección }\end{array}$ & $\begin{array}{l}\text { Con } \\
\text { tratamiento } \\
\text { convencional }\end{array}$ & $\begin{array}{l}\text { Con } \\
\text { tratamiento } \\
\text { avanzado }\end{array}$ & No utilizable & No utilizable \\
\hline $\begin{array}{l}\text { Abastecimiento de agua para actividades industri- } \\
\text { ales destinadas a la producción de alimentos de } \\
\text { consumo humano }\end{array}$ & $\begin{array}{l}\text { Sin tratamiento } \\
\text { previo o con } \\
\text { tratamiento } \\
\text { simple de } \\
\text { desinfección }\end{array}$ & $\begin{array}{l}\text { Con } \\
\text { tratamiento } \\
\text { convencional }\end{array}$ & $\begin{array}{l}\text { Con } \\
\text { tratamiento } \\
\text { avanzado }\end{array}$ & No utilizable & No utilizable \\
\hline $\begin{array}{l}\text { Abastecimiento de agua para abrevadero y ac- } \\
\text { tividades pecuarias }\end{array}$ & Sin limitaciones & Sin limitaciones & Sin limitaciones & $\begin{array}{l}\text { Con limita- } \\
\text { ciones }\end{array}$ & No utilizable \\
\hline Actividades recreativas de contacto primario & Utilizable & Utilizable & No utilizable & No utilizable & No utilizable \\
\hline Acuacultura & Utilizable & Utilizable & No utilizable & No utilizable & No utilizable \\
\hline $\begin{array}{l}\text { Fuente para la conservación del equilibrio natural } \\
\text { de las comunidades acuáticas }\end{array}$ & Utilizable & No utilizable & No utilizable & No utilizable & No utilizable \\
\hline $\begin{array}{l}\text { Fuente para la protección de las comunidades } \\
\text { acuáticas }\end{array}$ & Utilizable & Utilizable & No utilizable & No utilizable & No utilizable \\
\hline Generación hidroeléctrica & Utilizable & Utilizable & Utilizable & $\begin{array}{l}\text { Utilizable con } \\
\text { limitaciones }\end{array}$ & $\begin{array}{l}\text { Utilizable con } \\
\text { limitaciones }\end{array}$ \\
\hline Navegación & No utilizable & No utilizable & Utilizable & utilizable & Utilizable \\
\hline $\begin{array}{l}\text { Riego de especies arbóreas, cereales y plantas } \\
\text { forrajeras. }\end{array}$ & Utilizable & Utilizable & Utilizable & No utilizable & No utilizable \\
\hline $\begin{array}{l}\text { Riego de plantas sin limitación, irrigación de hor- } \\
\text { talizas que se consumen crudas o de frutas que } \\
\text { son ingeridas sin eliminación de la cáscara. }\end{array}$ & Utilizable & Utilizable & No utilizable & No utilizable & No utilizable \\
\hline
\end{tabular}


muchas variables o indicadores, que se transforman en una sola variable fácil de entender e interpretar. Esa facultad de los índices de cuantificar, simplificar y comunicar datos complejos es lo que los ha hecho un medio efectivo para comunicar información (CEPIS, 2008; Parparov, 2006). Para que un índice sea práctico, debe reducir una cantidad grande de indicadores a una forma más simple (León, 1992). Además, permite un mejor entendimiento de los asuntos referentes a la calidad del agua por parte del público y los tomadores de decisiones (Cude, 200 la).

El primer índice fue propuesto por Horton en 1965 (fórmula de cálculo I) y desde entonces se han desarrollado varios índices, que se diferencian entre sí en cuanto a los indicadores que los conforman y la expresión matemática para su cálculo (Cube, 200 I; Nasirian, 2007).

$$
I C A=\sum_{i=1}^{n} S l i * W i
$$

donde:

ICA es el índice de calidad del agua

Sli es el subíndice de cada indicador

Wi es el valor de ponderación para cada indicador según su importancia

Algunos índices representan una función lineal, otros son del tipo exponencial, otros son funciones de poder, etc. (Cude, 200 la,b). Usualmente los criterios empleados para la inclusión de los indicadores de calidad, utilizados en el cálculo de un índice, han sido básicamente subjetivos. León (1992), durante sus investigaciones en algunos ríos de México, propuso, apoyado en consultas a expertos, el uso de algunos indicadores para el cálculo de un índice. Entre ellos se mencionan el oxígeno disuelto, la demanda bioquímica de oxígeno, el pH, los sólidos suspendidos totales, los coliformes fecales, los nitratos, el amoníaco, los fosfatos, los fenoles, la alcalinidad, la dureza y los cloruros. Los índices requieren del cálculo de un subíndice (SI) y en ocasiones un nivel de ponderación para cada indicador (Wi). El SI transforma la medición absoluta de cada indicador, que muestran magnitudes y unidades de medición diversas, en un valor adimensional en una escala de 0 a I00. Valores de $\mathrm{SI}$ que tienden al 0 implican niveles de contaminación del agua cada vez mayores. En la literatura se mencionan distintas expresiones matemáticas que detallan en forma clara y precisa la forma de calcularlos (Abbasi, 2002, Cude, 200 Ia, Nasirian, 2007).

El citado reglamento costarricense contempla el uso de dos índices: el Índice Holandés de Valoración de la Calidad del agua del río y el índice biológico. El primero se basa en el análisis de tres parámetros fisicoquímicos y el segundo en el análisis de la fauna bentónica. El Indice Holandés emplea únicamente tres indicadores que son importantes para medir la calidad del agua, pero descartando así el uso de otros que son frecuentemente mencionados por otros investigadores como importantes. Tal es el caso de los coliformes fecales, relacionados con la contaminación humana y animal; o la presencia de fosfatos, fuertemente asociada en nuestros ríos a la contaminación antropogénica por la descarga de detergentes en aguas residuales de uso doméstico, solo para mencionar dos.

Con respecto al uso de estos índices y su relación con la clase-uso del agua, es importante mencionar que el índice basado en la presencia de fauna bentónica clasifica la calidad del agua en seis categorías diferentes sin que se establezca su relación con un uso o clase particular de los indicados en el reglamento. Por otra parte, el Indice Holandés clasifica el agua en cinco categorías de calidad distintas que a su vez la correlaciona con las clases mencionadas en el reglamento.

León (1992) y otros autores prefieren clasificar la calidad del agua según su grado de contaminación, para cada uso particular. Usualmente esta clasificación se efectúa asociando colores distintos a diferentes rangos de contaminación (Cube, 200la; León, 1992) Además, el reglamento incluye un número importante de indicadores físicoquímicos y microbiológicos complementarios, con sus respectivos rangos de permisibilidad para cada clase de agua de acuerdo con su concentración y que no son contemplados por los índices mencionados. El uso del Índice Holandés, basado únicamente en tres indicadores físicoquímicos, puede resultar en algún grado de ambigüedad con respecto a otros indicadores y en una falta de amplitud al momento de evaluar la calidad de un agua en particular. Si bien nuestra legislación hace bien en empezar a proponer herramientas útiles para medir el grado de contaminación de las aguas superficiales en forma fácil de analizar y entender, es importante considerar el uso de otros índices que se mencionan con 
frecuencia en la literatura y que involucran una mayor cantidad de indicadores bastante relevantes.

La metodología explicada en el reglamento es únicamente para el uso de los dos índices mencionados. La incorporación de otros indicadores como elementos de juicio para medir la calidad de las aguas es un aspecto importante que contempla nuestra legislación. Sin embargo, no explica cómo hacerlo ni tampoco la metodología que se debe utilizar para usar otros índices que se mencionan frecuentemente en la literatura con el respectivo empleo de una mayor cantidad de indicadores.

El objetivo de esta investigación se enfocó en verificar la aplicabilidad de fórmulas matemáticas para el cálculo del SI mencionadas en la literatura y su posible aplicación en Costa Rica, considerando nuestro entorno ambiental y la legislación nacional.

\section{Metodología}

El reglamento costarricense clasifica la calidad del agua en cinco clases diferentes que a su vez se relacionan con II usos distintos del agua (ver cuadro I). El análisis de cada uno de estos usos para cada clase particular conduciría a una investigación demasiado extensa. Resulta más práctico seleccionar una sola clase, independientemente de su uso, pero que se considere de alta relevancia. La Clase 2 del reglamento es una categoría altamente deseable en nuestros ríos ya que se asocia con actividades recreativas de contacto primario, con la acuacultura, así como con la protección de las comunidades acuáticas. Igualmente, esta calidad de agua se puede emplear en el riego de hortalizas que se consumen crudas o de frutas que se ingieren sin eliminación de la cáscara (MINAE, 2007). El agua para consumo humano se descarta como agua de interés en este estudio, ya que su manejo se hace a nivel de naciente y se recomienda protegerla por medio de una infraestructura apropiada que impida que prácticas antropogénicas, así como aspectos ambientales, repercutan en forma nociva sobre la calidad del líquido para el consumo humano.

Para el cálculo del SI se utilizaron las fórmulas de cálculo mencionadas por Cude, Nasiriam, Dinius, Prakash, Prati, Walski-Parker y Stoner (Abbassi, 2002, Cude, 200 Ia, Nasirian, 2007). Para verificar el comportamiento del SI para un indicador dado se efectuó el cálculo de cada SI con relación a distintas concentraciones que pudiera adoptar ese indicador. El rango apropiado de trabajo se obtuvo de los valores de permisibilidad aportados por el reglamento. Los distintos SI así generados deberían mostrar un comportamiento que varíe en una escala de 0 a 100. En general, a mayor concentración de un indicador en particular, mayor la tendencia del SI hacia 0, lo cual a su vez también debe mostrar una relación estrecha con los niveles de permisibilidad reportados en nuestro reglamento para cada indicador. Información parcial de estos datos de permisibilidad se muestra en el cuadro 3, pero para mayor información se recomienda ir directamente al reglamento nacional.

En aquellos casos en los cuales el comportamiento de la curva no se aplicaba a nuestro país, se propuso una nueva fórmula de cálculo. Para la elaboración de nuevas propuestas de cálculo del SI se generaron curvas de mejor ajuste. Su obtención se hizo con la ayuda del programa de cómputo CurveExpert Version 1.34. Para generar estas curvas, se asignó arbitrariamente un valor de $\mathrm{SI}=100$ para el valor de permisibilidad inferior de la clase I para cada indicador, un SI=90 para el valor superior de la clase I, un $\mathrm{SI}=80$ para el límite superior de la clase 2 y un valor $\mathrm{SI}=20$ para el nivel inferior de la clase 5 , tal como se muestra en el cuadro 3.

Igualmente fue necesario proponer la siguiente escala para clasificar la calidad del agua para su uso como clase 2, de acuerdo al valor del SI o del ICA y codificado, además, en colores. Fue necesario usar esta escala por colores para poder interpretar, en forma sencilla, los resultados que se obtengan en el empleo de alguno de los SI o los índices de calidad reportados en la literatura. Por otra parte, esta escala sirvió de base para el cálculo de las nuevas fórmulas de cada indicador físicoquímico y microbiológico, en aquellos casos en los que las fórmulas de cálculo propuestas por autores de otros países con características ambientales y regulatorias disímiles a las del nuestro hagan necesaria una propuesta de cálculo diferente.

\section{Resultados y discusión}

Al analizar las distintas fórmulas de cálculo propuestas por varios investigadores, se encontró que la mayoría de ellas son propias para las zonas a las cuales hacen referencia sus autores. Algunas son 
Figura I. Nueva propuesta para la clasificación de la calidad del agua para su uso como clase 2, según el valor del ICA o el SI y codificado en colores.

\section{NIVELES DE CALIDAD DEL AGUA}

\section{Clase 2}

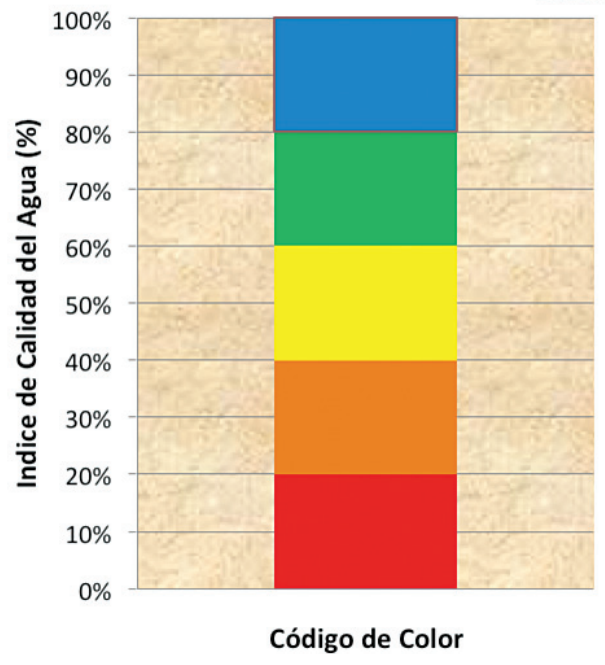

\section{Excelente Calidad pasa su} uso en clase 2

Contaminación Incipiente

\section{Contaminación Moderada}

- Contaminación Severa

- Contaminación Muy Severa

Cuadro 2. Fórmulas de cálculo propuestas por distintos investigadores para la determinación del subíndice (SI) para varios indicadores de la calidad del agua

\begin{tabular}{|c|c|c|c|}
\hline Indicador & Autor & \multicolumn{2}{|c|}{ Fórmula } \\
\hline Oxígeno disuelto & Cude & $\begin{array}{l}S I=10 \\
S I=-80.29+31.88 * X-1.401 * X^{2} \\
S I=100\end{array}$ & $\begin{array}{l}\text { para } X \leq 3.3 \\
\text { para } 3.3<X<10.5 \\
\text { para } 10.5 \leq X\end{array}$ \\
\hline Oxígeno disuelto & Wolski-Parker & $\begin{array}{l}S I=e^{0.3 *(X-8)} \\
S I=0\end{array}$ & $\begin{array}{l}\text { para } 0<X \leq 8 \\
\text { para } 8<X\end{array}$ \\
\hline $\begin{array}{l}\text { Porcentaje de saturación de } \\
\text { oxígeno }\end{array}$ & Nasirian & $\begin{array}{l}S I=100 \\
S I=-0,00020 X^{3}+0,030 X^{2}-0,395 \\
S I=0\end{array}$ & $\begin{array}{l}\text { para } X \geq 92 \\
\text { para } 8<X<92 \\
\text { para } X \leq 8,0 \text { y } X>140\end{array}$ \\
\hline $\begin{array}{l}\text { Porcentaje de saturación de } \\
\text { oxígeno }\end{array}$ & Prakash & $\begin{array}{l}S I=0.18+0.66 \\
S I=-13.5+1.17 \\
S I=263.34-0.62\end{array}$ & $\begin{array}{ll}\text { para } & 0-40 \% \\
\text { para } & 40-100 \% \\
\text { para } & 100-140 \%\end{array}$ \\
\hline $\begin{array}{l}\text { Porcentaje de saturación de } \\
\text { oxígeno }\end{array}$ & Prati & $\begin{array}{l}\mathrm{SI}=0.00168 * X^{2}-0.249 * X+12.25 \\
\mathrm{SI}=-0.08 * X+8 \\
\mathrm{SI}=0.08 * X-8\end{array}$ & $\begin{array}{ll}\text { para } & 0 \leq X<50 \\
\text { para } & 50 \leq X<100 \\
\text { para } & 100 \leq X\end{array}$ \\
\hline $\begin{array}{l}\text { Porcentaje de saturación de } \\
\text { oxígeno }\end{array}$ & Dinius & $S I=X$ & \\
\hline $\begin{array}{l}\text { Demanda bioquímica de } \\
\text { oxígeno }\end{array}$ & Dinius & $\begin{array}{l}S I=100 \\
S I=+108 X^{-0,3494}\end{array}$ & para $X \leq 1,25$ \\
\hline $\begin{array}{l}\text { Demanda bioquímica de } \\
\text { oxígeno }\end{array}$ & Dinius & $S I=107 * X^{-0.642}$ & \\
\hline $\mathrm{pH}$ & Cude & $\begin{array}{l}\mathrm{SI}=10 \\
\mathrm{SI}=+2,628 \mathrm{e}^{+0,5200 \times} \\
\mathrm{SI}=100 \\
\mathrm{SI}=100 \mathrm{e}^{-0,5188(X-8)} \\
\mathrm{SI}=10\end{array}$ & $\begin{array}{l}\text { para } X<4 \\
\text { para } 4 \leq X<7 \\
\text { para } 7 \leq X \leq 8,0 \\
\text { para } 8<X \leq 11,0 \\
\text { para } X>||\end{array}$ \\
\hline
\end{tabular}


Continuación

\begin{tabular}{|c|c|c|c|}
\hline Indicador & Autor & \multicolumn{2}{|l|}{ Fórmula } \\
\hline $\mathrm{pH}$ & Nasirian & $\begin{array}{l}\mathrm{SI}=0 \\
\mathrm{SI}=0 \\
\mathrm{SI}=17.2-17.2 * X+5.02 * X^{2} \\
\mathrm{SI}=-248+95.5 * X-6.67 * X^{2} \\
\mathrm{SI}=-18 I+82.4 * X-6.05 * X^{2} \\
\mathrm{SI}=536-77.0 * X+2.76 * X^{2}\end{array}$ & $\begin{array}{l}\text { para } 0<X \leq 2 \\
\text { para } X \geq 12 \\
\text { para } 2<X<5.5 \\
\text { para } 5.5<X<7 \\
\text { para } 7<X<8.75 \\
\text { para } 8.75<X<12\end{array}$ \\
\hline Sólidos suspendidos totales & Prati & $S I=2^{(2.1 * \log (0.1 * X-1)}$ & \\
\hline Sólidos suspendidos totales & Walski-Parker & $\mathrm{SI}=\mathrm{e}^{(-0.02 * X) * 100}$ & \\
\hline Sólidos totales & Cude & $\begin{array}{l}\mathrm{SI}=100 \\
\mathrm{SI}=10 \\
\mathrm{SI}=142.6 * \mathrm{e}^{-0.00886^{*} \times}\end{array}$ & $\begin{array}{l}\text { para } X \leq 40 \\
\text { para } X>220 \\
\text { para } 40<x \leq 220\end{array}$ \\
\hline Coliformes fecales & Dinius & $\mathrm{SI}=100 *(5 * X)^{-0.3}$ & \\
\hline Coliformes fecales & Stoner & $S I=100-0.000025 * X^{2}$ & \\
\hline Nitrógeno amoniacal & Stoner & $S I=100-100 * X^{2}$ & \\
\hline Amoníaco & Prati & $\mathrm{SI}=2^{\left(2.1 * \log \left(10^{*} \times\right)\right.}$ & \\
\hline Fosfatos & Nasirian & $\begin{array}{l}S I=2 \\
S I=0.047 * X^{4}-1.338 * X^{3}+13.276 * X^{2}-55.247 * X\end{array}$ & $\begin{aligned} & \text { para } X>10 \\
x+94.439 & \text { para } X \leq 10\end{aligned}$ \\
\hline Fosfatos & Walski-Parker & $S I=e^{-2.5 * x}$ & \\
\hline Alcalinidad & Dinius & $S I=110 X^{-0.1342}$ & \\
\hline Dureza & Dinius & $\mathrm{SI}=1 \mathrm{O}^{(+1,974-0,00132 X)}$ & \\
\hline Cloruros & Prati & $\begin{array}{l}\mathrm{SI}=0.000228 * X^{2}+0.0314 * X \\
\mathrm{SI}=0.000132 * X^{2}+0.0074 * X+0.6 \\
\mathrm{SI}=3.75 *(0.02 * X-5.2) * 5.2\end{array}$ & $\begin{array}{l}\text { para } 0 \leq X<50 \\
\text { para } 50 \leq X<300 \\
\text { para } 300 \leq X\end{array}$ \\
\hline Cloruros & Dinius & $\begin{array}{l}\mathrm{SI}=125.8 * X^{-0.207} \\
\mathrm{SI}=100\end{array}$ & para $X \leq 3.0$ \\
\hline Cloruros & Dinius & $\begin{array}{l}\mathrm{SI}=39 \mid * \mathrm{X}^{-0.34807} \\
\mathrm{SI}=100\end{array}$ & para $X \leq 50.0$ \\
\hline Cloruros & Stoner & $\begin{array}{l}S I=100 \\
S I=-0.0005 * X^{2}-0.0125 * X+98.296\end{array}$ & para $X=0$ \\
\hline Sulfatos & Nasirian & $\begin{array}{l}S I=-0, I X+100 \\
S I=0\end{array}$ & $\begin{array}{l}\text { para } X<1000 \\
\text { para } X \geq 1000\end{array}$ \\
\hline Conductividad & Stoner & $\begin{array}{l}S I=+100-0,0002 X^{2} \\
S I=2\end{array}$ & $\begin{array}{l}\text { para } X<700 \\
\text { para } X \geq 700\end{array}$ \\
\hline Fenoles & Stoner & $\begin{array}{l}S I=+100-100 X \\
S I=0\end{array}$ & $\begin{array}{l}\text { para } X<1,0 \\
\text { para } X \geq 1\end{array}$ \\
\hline Turbiedad & Walski-Parker & $S I=e^{-0.001 * X}$ & \\
\hline
\end{tabular}

para usos diferentes a las aguas superficiales $y$, además, los valores de permisibilidad empleados son diferentes a los indicados por nuestra legislación, lo cual las hace inviables para su uso en Costa Rica. Por ejemplo, en el caso del contenido de cloruros para la clase 2, nuestra reglamentación establece un rango de 100-200 mg/L y utilizando la clasificación propuesta en la Figura I, este rango debería dar un valor de $\mathrm{SI} \geq 80 \%$ y, por lo tanto, clasificarse como de excelente calidad para su uso en clase 2.
Utilizando las fórmulas de cálculo para este indicador dadas por Nasirian y Stoner (ver cuadro 2), los SI corresponden a valores cercanos a 100\%; por otra parte, el uso de una de las fórmulas de cálculo de Dinius genera valores de SI cercanos al 50\%; mientras que otra de las fórmulas de este mismo autor para este indicador da valores de SI cercanos a $0 \%$. Asimismo, la fórmula de cálculo recomendada por Stoner genera valores negativos para concentraciones de cloruros superiores a los $800 \mathrm{mg} / \mathrm{L}$, lo 
cual carece de sentido físico. Claramente los valores calculados, en este caso particular, utilizando las fórmulas propuestas por otros investigadores, distan de ser similares a lo esperado.

Otro caso importante de mencionar es la fórmula de cálculo del SI para el contenido de oxígeno disuelto (OD) que presenta Cude. Un contenido de oxígeno disuelto (OD) que represente un $\mathrm{SI}=100 \%$ empleando su fórmula de calculo corresponde a un $O D=11,0 \mathrm{mg}$ de oxígeno/L, pero como el contenido de oxígeno disuelto en el agua depende de la temperatura, este valor se obtendría si la temperatura del agua fuera de $11{ }^{\circ} \mathrm{C}$, en un nivel de saturación y una presión atmosférica de 101.3 $\mathrm{KPa}$. La temperatura de las aguas de nuestros ríos usualmente es de $(20-25)^{\circ} \mathrm{C}$, lo que corresponde a valores de $O D=(7,0-7,7) \mathrm{mg} / \mathrm{L}$ a 1400 metros de altitud. Nuevamente se tiene una situación en la que la fórmula de cálculo propuesta por Cude no se aplica a las condiciones ambientales de nuestro país. Un aspecto importante de considerar es que el valor de 5,0 mg de oxígeno disuelto/L es crítico, ya que valores por debajo de este dato dificultan la vida de los peces en los ríos. Esta consideración se contempló para la propuesta de una nueva fórmula de cálculo del OD (ver cuadro 4).
De esta forma, se evaluaron varias fórmulas de cálculo para cada uno de los indicadores mostrados en el cuadro 2. Entre las fórmulas reportadas por otros autores, son pocas las que se ajustan a las características propias de nuestro país. En la mayoría de los casos se propuso una nueva fórmula de cálculo que considera las condiciones ambientales de Costa Rica, así como su reglamentación y los niveles de contaminación que históricamente han mostrado sus ríos. Las fórmulas de cálculo propuestas que se indican en el cuadro 4 son para el uso del agua de clase 2 y para los niveles de permisibilidad vigentes de nuestra legislación.

\section{Conclusiones}

El reglamento para la clasificación y evaluación de la calidad de los cuerpos de agua superficiales de Costa Rica nos ofrece varias herramientas para valorar la calidad de nuestros ríos. Si bien menciona una serie de indicadores físicoquímicos, microbiológicos y biológicos importantes, solo hace referencia a dos índices para medir esta calidad, siendo uno de ellos el Índice Holandés, que es el único que establece alguna correlación con las cinco distintas clases mencionadas en el reglamento. Al basarse solamente en tres indicadores, se corre el riesgo de caer en la ambigüedad y falta de solidez en

Cuadro 3. Niveles de concentración permitidos para algunos de los indicadores que forman parte del reglamento, según cada clase de calidad del agua en el reglamento.

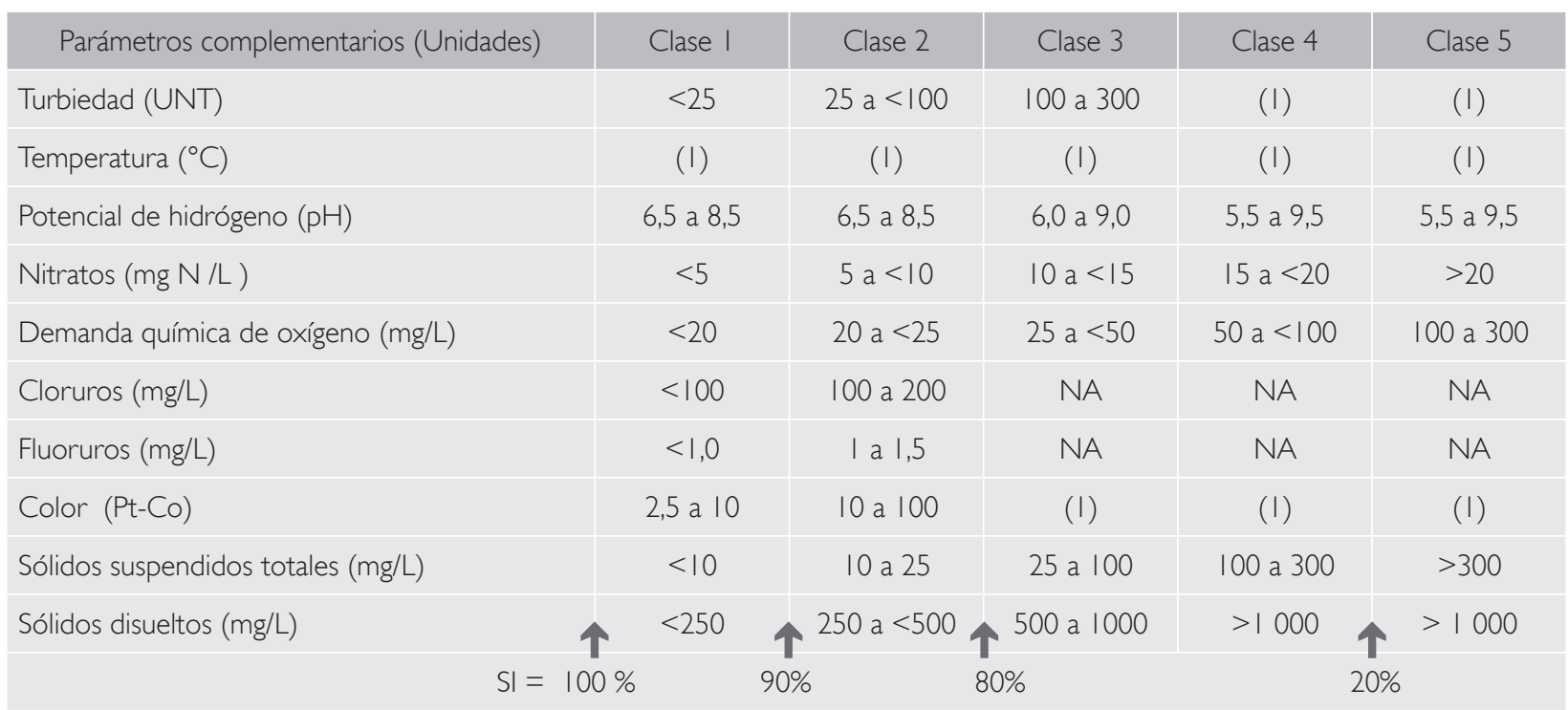

Fuente: La Gaceta, Diario Oficial, \# | 78, 2007 
Figura 2. Comportamiento de las fórmulas de cálculo del SI utilizando las fórmulas propuestas por varios autores

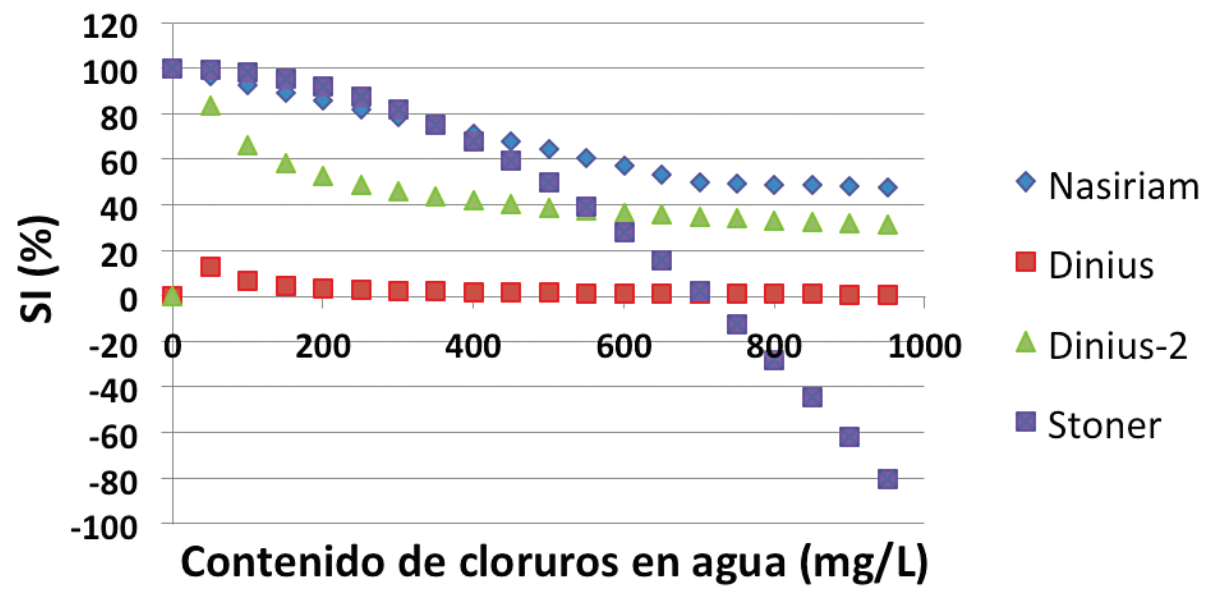

Cuadro 4. Fórmulas para el cálculo del subíndice (Sli) para varios indicadores de la calidad del agua

\begin{tabular}{|c|c|c|}
\hline Indicador & Autor & Fórmula \\
\hline Oxígeno disuelto & Propuesta nueva & $\mathrm{SI}=\frac{a}{1+b e^{-c x}} \quad$ donde $\mathrm{a}=97,080537, \mathrm{~b}=60.4005|5, \mathrm{c}=1,| \mathrm{|} 80264$ \\
\hline $\begin{array}{l}\text { Porcentaje de } \\
\text { saturación de } \\
\text { oxígeno }\end{array}$ & Propuesta nueva & $\mathrm{SI}=\frac{a}{1+b e^{-c x}} \quad$ donde $\mathrm{a}=|0| .|40|, b=48.05573, c=0.0817 \mid 4$ \\
\hline $\begin{array}{l}\text { Demanda bioquímica } \\
\text { de oxígeno }\end{array}$ & Propuesta nueva & $\begin{array}{l}\mathrm{SI}=\frac{1}{a+b x^{c}} \\
\text { donde } \mathrm{a}=0,010027, \mathrm{~b}=0,000352, \mathrm{c}=1,735 \mid 42\end{array}$ \\
\hline $\mathrm{pH}$ & Cude & 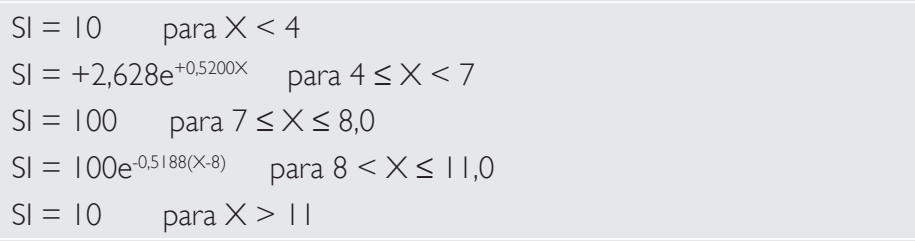 \\
\hline $\begin{array}{l}\text { Sólidos suspendidos } \\
\text { totales }\end{array}$ & Propuesta nueva & $\begin{aligned} \mathrm{SI} & =100 \text { para } \mathrm{X} \leq \mathrm{I} \\
\mathrm{SI} & =\frac{1}{a+b x^{c}} \text { donde } \mathrm{a}=0,009946, \mathrm{~b}=0,0000826, \mathrm{c}=1, \mathrm{I} \mid \mathrm{O} 0736\end{aligned}$ \\
\hline Sólidos totales & Propuesta nueva & $\begin{array}{l}S I=0 \text { para } X \geq 1400 \\
S I=100 \text { para } X \leq 1 \\
S I=a+b x+c x^{2}+d x^{3} \quad \text { donde } a=|00.5| 45, b=-0.06723, c=0,0000969 \\
d=-7.12 * 10^{-8}\end{array}$ \\
\hline Coliformes fecales & Propuesta nueva & $\begin{array}{l}\mathrm{SI}=0 \text { para } X \geq 9000 \\
\mathrm{SI}=\mathrm{a}+\mathrm{bx}+c x^{2} \quad \text { donde } \mathrm{a}=96,42218, \mathrm{~b}=-0,0195, \mathrm{c}=9,80 * 10^{-07}\end{array}$ \\
\hline Nitratos & Propuesta nueva & $\begin{array}{l}\mathrm{SI}=0 \quad \text { para } \mathrm{X} \geq 22.4 \\
\mathrm{SI}=\mathrm{a}+\mathrm{bx}+\mathrm{cx^{2 }} \quad \text { donde } \mathrm{a}=99,42857, \mathrm{~b}=-0,57|43, \mathrm{c}=-0,17| 43\end{array}$ \\
\hline Nitrógeno amoniacal & Propuesta nueva & $\mathrm{SI}=\frac{a}{1+b e^{-c x}} \quad$ donde $\mathrm{a}=-|24,008, \mathrm{~b}=-2,24954, \mathrm{c}=-0,2276|$ \\
\hline
\end{tabular}


Continuación

\begin{tabular}{|c|c|c|}
\hline Indicador & Autor & Fórmula \\
\hline Amoníaco & Propuesta nueva & $\mathrm{SI}=\frac{a}{1+b e^{-c x}} \quad$ donde $\mathrm{a}=-124,009, \mathrm{~b}=-2,24955, \mathrm{c}=-0,18744$ \\
\hline Fósforo de fosfatos & Propuesta nueva & $\mathrm{SI}=\frac{1}{\left(a+b x+c x^{2}\right)} \quad$ donde $\mathrm{a}=0,01012, \mathrm{~b}=0,020003, \mathrm{c}=0,009649$ \\
\hline Fosfatos & Propuesta nueva & $\mathrm{SI}=\frac{a}{1+b e^{-c x}} \quad$ donde $\mathrm{a}=-48,6896, \mathrm{~b}=-\mathrm{I}, 496 \mid 4, \mathrm{c}=-0,19392$ \\
\hline Alcalinidad & Propuesta nueva & $\begin{array}{l}\mathrm{SI}=100 \text { para } \mathrm{X} \geq 31.9 \\
\mathrm{SI}=\frac{a * b+c * x^{d}}{b+x^{d}} \quad \text { donde } \mathrm{a}=-1.63^{*} 10^{-11}, \mathrm{~b}=20,10495 \\
\mathrm{c}=263,2578, \mathrm{~d}=0,725064\end{array}$ \\
\hline Dureza & Dinius & $S I=10^{(+1,974-0,00132 X)}$ \\
\hline Cloruros & Propuesta nueva & $\begin{array}{l}\mathrm{SI}=\mathrm{a}+\mathrm{b} \times \quad \text { donde } \mathrm{a}=100, \mathrm{~b}=-0.1 \\
\mathrm{SI}=0 \text { para } X \geq 1000\end{array}$ \\
\hline Sulfatos & Propuesta nueva & $\begin{array}{l}\mathrm{SI}=\mathrm{a}+\mathrm{bx}+\mathrm{cx^{2 }} \text { donde } \mathrm{a}=100, \mathrm{~b}=-0,04667, \mathrm{c}=-0,00013 \\
\mathrm{SI}=0 \quad \text { para } \mathrm{X}>708\end{array}$ \\
\hline Conductividad & Stoner & $\begin{array}{l}S I=+100-0,0002 X^{2} \quad \text { para } X<700 \\
S I=0 \text { para } X \geq 700\end{array}$ \\
\hline Fenoles & Stoner & $\begin{array}{l}\mathrm{SI}=+100-100 X \quad \text { para } X<1,0 \\
\mathrm{SI}=0 \quad \text { para } X \geq 1\end{array}$ \\
\hline Turbiedad & Propuesta nueva & $\begin{array}{l}\mathrm{SI}=\mathrm{a}+\mathrm{bx}+\mathrm{cx^{2 }} \text { donde } \mathrm{a}=97,50282, \mathrm{~b}=-0,18567, \mathrm{c}=0,0000882 \\
\mathrm{SI}=0 \quad \text { para } \mathrm{X}>1000\end{array}$ \\
\hline
\end{tabular}

el modelo de cálculo empleado y su interpretación. Por lo tanto, es conveniente el uso de otros índices de calidad que consideran un número mayor de indicadores para su cálculo, lo que genera un modelo más robusto y confiable. Es en este sentido que la información suministrada en el cuadro 4 y la Figura I se vuelve útil para el cálculo de otros índices mencionados en la literatura que usualmente requieren de un mayor número de indicadores.

La clase 2 es de especial importancia como referencia de la calidad que es deseable en nuestros ríos. En mi opinión, la calidad buscada en un río debe ser aquella que preserve la fauna acuática, permita su uso para actividades agroindustriales como el lavado de hortalizas y frutas que se consumen crudas, así como el uso recreativo de tipo primario. De esta forma, se establece un punto de partida para evaluar la calidad de los ríos del país. El uso del agua para consumo humano implica un manejo y protección a nivel de nacientes o de aguas subterráneas en nuestro país, por lo cual se hace necesario enfocarlo desde otro punto de vista.

\section{Agradecimientos}

Este artículo forma parte del tema de tesis del estudiante de doctorado Guillermo Calvo Brenes, del Sistema de Estudios de Posgrado del Programa Doctorado en Ciencias Naturales para el Desarrollo (DOCINADE).

\section{Bibliografía}

Abbasi, S.A. (2002). Water quality indices. Roorkee, India: INCOH Secretariat, National Institute of Hydrology.

CEPIS (2008). Modelos matemáticos de calidad del agua. Extraído el 30 de septiembre de 2008 desde http: www.cepis.org.pe/ bvsaca/e/modemate.html

Cude, C.G. (200Ia). Oregon water quality index: a tool for evaluating water quality management effectiveness. Journal of the American Water Resources Association 37( I), 125- 137.

Cude, C.G. (200 lb). The Oregon water quality index (OWQI): A communicator of water quality information. Work presented in Spring Specialty Conference Proceedings, American Resources Association, Middleburg, Virginia, USA, TPS-0 I - I.

Department of Environmental Conservation (201I). Water quality standards. Chapter 70. Alaska. Extraído el 2 de 
febrero de 2012 desde http://dec.alaska.gov/water/wqsar/ wqs/pdfs/70mas.pdf

Enderlein, S.; Enderlein, R.E. and Williams, W.P. (20I0). Water quality requirements. Chapter 2. Extraído el 2 de febrero de 2012 desde www.who.int/water_sanitation_health/ resourcesquality/wpcchap2.pdf

EPA (1988). Quality standards criteria summaries: a compilation of state/federal criteria. Office of Water Regulations and Standards. Washington. Extraído el 2 de febrero de 2012 desde http://yosemite.epa.gov/water/owrccatalog.nsf/e673c 95b | | 602f2385256ae I007279fe/8cbb4579 | 85c75d28525 6b06007259ac!OpenDocument.

EPA (2008). Surface water quality standards of the Florida Administrative Code. Chapter 62-302. United States Environmental Protection Agency. Extraído el 2 de febrero de 2012 desde www.Water.epa.gov/scitech/swguidance/ standards/upload/2008_12_29_Standards_wqslibrary_fl_ fl_4_62-302.pdf

EPA (20 I2). Quality criteria for water. EPA-440/9-76-023. United States Environmental Protection Agency. Extraído el 2 de febrero de 2012 desde www.water.epa.gov/scitech/swguidance/standards/criteria/current/index.cfm

FAO (200 I). Indicadores de la calidad de la tierra y su uso para la agricultura sostenible y el desarrollo rural. Boletín de tierras y aguas de la FAO. Extraído el 15 de junio de 2009 desde http://uned.blackboard.com/webct/urw/lc5 I |600 I. tp0/cobaltMainFrame.dowebct
León, L. (1992). Indices de calidad del agua (ICA), forma de estimarlos y aplicación en la Cuenca Lerma-Chapala. Trabajos presentados en el VIII Congreso Nacional: Acciones para un ambiente limpio. Instituto Mexicano de Tecnología del Agua. Extraído el I de noviembre del 2009 desde http://www. science.uwaterlooca/ /fleonvi/rtic/art09.pdf.

MINAE (2007). Reglamento para la clasificación y la evaluación de la calidad de cuerpos de agua superficiales. No 33903 MINAE-S. Gaceta \#178, 17 de septiembre de 2007. San José.

Nasirian, M. (2007). A new water quality index for environmental contamination contributed by mineral processing: a case study of amang (tin tailing) processing activity. Journal of applied sciences 7(20), 2977-2987.

Natural Resources Board (20ll). Vermont water quality standards. National Life Records Center. State of Vermont, USA. Extraído el 2 de febrero de 2012 desde http://www.hrb. state.vt.us/wrp.rules.htm

Parparov, A. Hambright, K.D.; Hakanson, L. and Ostapenia, A. (2006). Water quality quantification: basics and implementation. Hydrobiologia 560( I), p227-237.

Swarnee, P.K. \& Tyagi, A. (2007). Improved method for aggregation of water quality subindices. Journal of environmental engineering I33(2), p. 220-225. 